\title{
Light-Emitting Diode Degradation and Low-Frequency Noise Characteristics
}

\author{
B. Šaulys, J. Matukas, V. Palenskis, S. Pralgauskaité丶 $\dot{H}^{*}$ and G. Kulikauskas \\ Radiophysics Dep., Vilnius University, Saulètekio av. 9 (III), LT-10222 Vilnius, Lithuania
}

\begin{abstract}
Comprehensive investigation of phosphide-based red and nitride-based blue light-emitting diodes characteristics and physical processes that take place in device structure during aging has been carried out. Analysis of noise characteristics (the emitting-light power and the LED voltage fluctuations, also their cross-correlation factor) shows that investigated LEDs degradation is caused by defects that lead to the leakage current and non-radiating recombination increase in the active region or its interfaces. Appearance of the defects first of all manifests in noise characteristics: intensive and strongly correlated $1 / f^{\alpha}$ type optical and electrical fluctuations come out.
\end{abstract}

PACS: 72.70. $+\mathrm{m}, 74.40 .-\mathrm{n}, 85.30 .-\mathrm{z}, 85.60 . \mathrm{Jb}$

\section{Introduction}

Nowadays light-emitting diodes (LEDs) span all visible light spectrum and beyond. Different materials are used for the fabrication of the LEDs with different color of light: phosphide-based materials are used to cover infrared, red-yellow light spectrum regions, while for green-blue, ultraviolet regions, also white LEDs, nitride-based materials are common. All LED applications expect stable and long device operation. Thus, advances in LED quality and lifetime improvement are point of interest between manufacturers.

Understanding of physical processes that worsen the light-emitting diode quality and accelerate its degradation stimulates an improvement of LEDs fabrication technology. Degradation of LEDs usually is related with defects in device active region [1]. Nitride-based structures distinguish by high mechanical strength and durability, and have the potential to be used for high-temperature and power electronics [2-4]. But nitride layer is a source of a number of threading dislocations in the active region of LED originating from the interface between GaN and substrate due to lattice mismatch [5]. Presence of the defects leads to the injected electron-hole pairs capture and their nonradiative recombination that lowers the efficiency of the light-emitting device and accelerates device aging [6]. The question, in which part of the structure defects appear, how they influence the device operation characteristics and how they behave during device aging, is always actual for any semiconductor structure. At this point, noise investigation gives valuable information and is useful for evaluating semiconductor device quality and predicting lifetime [1, 7-11].

\footnotetext{
* corresponding author; e-mail: sandra.pralgauskaite@ff.vu.lt
}

Here we present comprehensive investigation of phosphide-based (AlInGaP) red and nitride-based (InGaN) blue light-emitting diodes operation (light output power and current-voltage) and noise (emitting-light power and the LED voltage fluctuations and their simultaneous cross-correlation factor) characteristics during device aging. The aim of the investigation was to clear up physical processes that take place during LEDs aging and clarify origin of their degradation.

\section{Experiment details}

High power $(1 \mathrm{~W})$ red LEDs radiating at $625 \mathrm{~nm}$ fabricated from AlInGaP and blue LEDs radiating at $455 \mathrm{~nm}$ based on InGaN have been investigated.

Optical, electrical and noise characteristics have been carried out at room temperature for initial samples and at defined time intervals during LEDs aging. Aging was performed at maximum permissible forward current $(350 \mathrm{~mA})$ at room temperature.

Optical characteristics (light output power) and optical noise (light output power fluctuations) of the LED have been measured by photodetector: the voltage of the photodetector load resistor is proportional to the LED output light power, and the measured photodetector voltage fluctuations correspond to the output light power fluctuations (optical noise). Electrical noise is the voltage fluctuations at LED terminals at constant forward current: the load resistance of LED was about 30-100 times larger than the differential resistance of LED, therefore current generator mode is guaranteed. All load resistors were tested in order to assure that they did not produce any additional current noise.

Noise characteristics have been measured under continuous wave operation and investigated in the frequency range from $10 \mathrm{~Hz}$ to $20 \mathrm{kHz}$. Optical and 
electrical noise signals were processed simultaneously using two identical channels comprised from low noise amplifiers, filter systems, analog digital converter (National Instrument ${ }^{\mathrm{TM}}$ PCI 6115 board) $[12,13]$. Such measurement technique allows us to measure the noise spectra and to calculate correlation between two noise sources. Noise intensity was evaluated by comparison with thermal noise of reference resistor $R_{\text {ref }}$ :

$$
S_{V}=\frac{\overline{V^{2}}-\overline{V_{\mathrm{s}}^{2}}}{\overline{V_{\text {ref }}^{2}}-\overline{V_{\mathrm{s}}^{2}}} 4 k T_{0} R_{\mathrm{ref}},
$$

where $\overline{V^{2}}, \overline{V_{\mathrm{s}}^{2}}$ and $\overline{V_{\text {ref }}^{2}}$ are the sample (optical or electrical) noise, the measuring system own noise, and the reference resistor thermal noise variances in the narrow frequency band $\Delta f ; T_{0}$ is the absolute temperature of the reference resistor. Correlation factor was defined by the following expression:

$$
r=\left\langle u_{\text {el total }}(t) u_{\text {ph total }}(t)\right\rangle /\left(\sigma_{\text {el total }}^{2} \sigma_{\text {ph total }}^{2}\right)^{1 / 2},
$$

where brackets $\langle\ldots\rangle$ mean averaging both on time and on number of realizations, and $\sigma_{\text {el total }}^{2}=\left\langle u_{\text {el total }}^{2}(t)\right\rangle$, $\sigma_{\mathrm{ph} \mathrm{total}}^{2}=\left\langle u_{\mathrm{ph} \text { total }}^{2}(t)\right\rangle$ are the total variances of electrical and optical fluctuations

$$
\begin{aligned}
& u_{\mathrm{el} \mathrm{total}}(t)=u_{\mathrm{el} 1 / f}(t)+u_{\mathrm{el} 1 / f^{\alpha}}(t)+u_{\mathrm{el} \mathrm{gr}}(t) \\
& \quad+u_{\mathrm{el} \mathrm{shot}}(t)+u_{\mathrm{el} \mathrm{syst}}(t), \\
& u_{\mathrm{ph} \mathrm{total}}(t)=u_{\mathrm{ph} 1 / f}(t)+u_{\mathrm{ph} 1 / f^{\alpha}}(t)+u_{\mathrm{ph} \mathrm{gr}}(t) \\
& \quad+u_{\mathrm{ph} \mathrm{shot}}(t)+u_{\mathrm{ph} \mathrm{syst}}(t),
\end{aligned}
$$

where $u_{\mathrm{el} 1 / f}(t)$ and $u_{\mathrm{ph} 1 / f}(t)$ are, respectively, the electrical and optical fluctuation components with $1 / f$ type spectrum; $u_{\mathrm{el} 1 / f^{\alpha}}(t)$ and $u_{\mathrm{ph} 1 / f^{\alpha}}(t)$ are, respectively, the electrical and optical fluctuation components with $1 / f^{\alpha}$ type spectrum; $u_{\mathrm{el} \mathrm{gr}}(t)$ and $u_{\mathrm{ph} \mathrm{gr}}(t)$ are, respectively, the electrical and optical fluctuation components with Lorentzian type spectrum; $u_{\mathrm{el}} \mathrm{sh}(t)$ and $u_{\mathrm{ph} \operatorname{sh}}(t)$ are, respectively, the electrical and optical fluctuation components with shot noise spectrum; $u_{\text {el syst }}(t)$ and $u_{\text {ph syst }}(t)$ are, respectively, the own noise components of electrical and optical noise measurement systems. The expression (2) allows one to obtain not only the magnitude of the cross-correlation factor, but also the correlation sign. Such presentation is very useful for further analysis of noise properties because it assumes that noise sources with $1 / f, 1 / f^{\alpha}$ and Lorentzian type spectra and shot noises are statistically independent.

A special attention was paid to correlation factor: simultaneous cross-correlation factor $r$ was measured not only over frequency range from $10 \mathrm{~Hz}$ to $20 \mathrm{kHz}$, but additionally in every one-octave frequency band

$$
\begin{gathered}
r_{\mathrm{oct}}=\left\langle u_{\mathrm{el} \mathrm{total} \mathrm{oct}}(t) u_{\mathrm{ph} \text { total oct }}(t)\right\rangle \\
/\left(\sigma_{\text {el total oct }}^{2} \sigma_{\mathrm{ph} \text { total oct }}^{2}\right)^{1 / 2},
\end{gathered}
$$

where the meanings of quantities are the same as in Eq. (2).

\section{Results and discussions}

\subsection{Noise spectra of investigated LEDs}

The spectra of fluctuations at low frequencies for investigated LEDs are mainly characterized by $1 / f^{\alpha}$-type electrical and optical noises both for phosphide-based and nitride-based devices (Figs. 1 and 2). This type of noise is due to many charge carriers generation-recombination (GR) processes in GR centers with widely distributed relaxation times [7]. These GR centers are formed by different defects and imperfections in the device structure, and can be located in the active region or other areas of the LED. The charge carrier capture and release processes in the active layers of the LED modulate that part of current which recombine in quantum wells and produce the photons and therefore lead to the optical $1 / f^{\alpha}$-type noise at low frequencies.

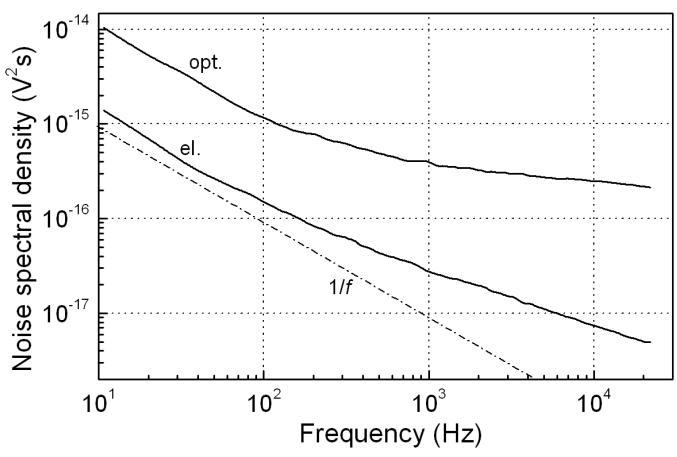

Fig. 1. Electrical (el.) and optical (opt.) noise spectra for blue LEDs before aging at $10 \mathrm{~mA}$ forward current.

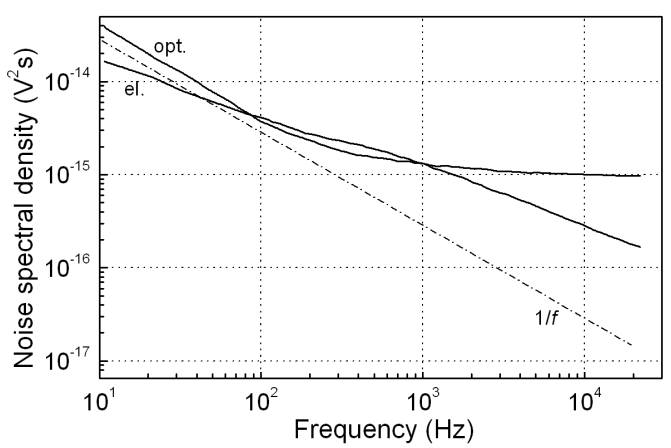

Fig. 2. Electrical (el.) and optical (opt.) noise spectra for red LEDs before aging at $10 \mathrm{~mA}$ forward current.

For LED optical fluctuation spectra there is a characteristic that at higher frequencies "white" noise prevails $1 / f^{\alpha}$-type fluctuations (Figs. 1 and 2). Optical fluctuations with "white" spectrum are caused by shot noise due to emitted photons.

The electrical noise level of the LEDs at low forward current region is large and decreases with forward current increasing. This intensive electrical noise at small 
currents is related with the current flow not through the whole device cross-section, but through the narrow channels formed by defects, and therefore, the random charge carrier recombination processes produce the intensive electrical noise at LED terminals. As current density increases the flow becomes more even through the cross-section and influence of separate defects decreases.

Optical noise intensity gradually increases with forward current increase due to light output power increasing.

\subsection{Aging process in InGaN blue LEDs}

Current-voltage $(I-V)$ and light output power vs. current $(L-I)$ characteristics of investigated nitride-based blue light LEDs are presented in Figs. 3 and 4, respectively.

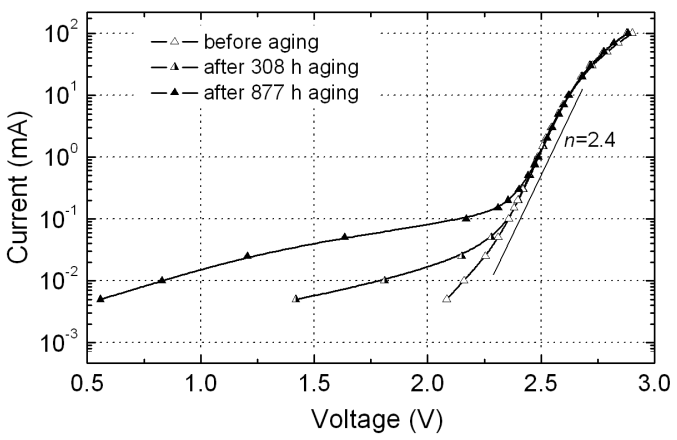

Fig. 3. Typical current-voltage characteristics of blue LED before (open symbols), after $308 \mathrm{~h}$ (half dark symbols) and $877 \mathrm{~h}$ (solid symbols) aging.

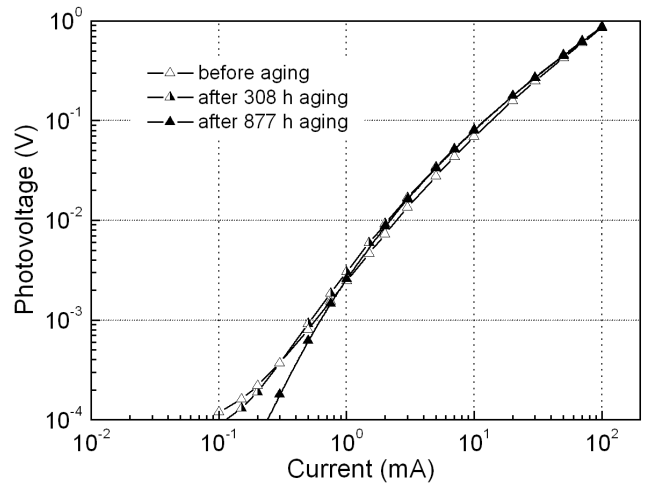

Fig. 4. Typical light output power vs. current characteristics of blue LED before (open symbols), after $308 \mathrm{~h}$ (half dark symbols) and $877 \mathrm{~h}$ (solid symbols) aging (the light output power is proportional to the voltage of photodetector load resistance).

$I-V$ characteristic of not aged device is typical for LEDs (Fig. 3): the non-ideality factor $n$ of the $I-V$ characteristic $\left(I=I_{0} \exp \left(e U_{\mathrm{d}} /(n k T)\right)\right)$ is about 2.4 in the current range from $0.1 \mathrm{~mA}$ to $10 \mathrm{~mA}$. Such $I-V$ characteristic indicates that current flow through the device

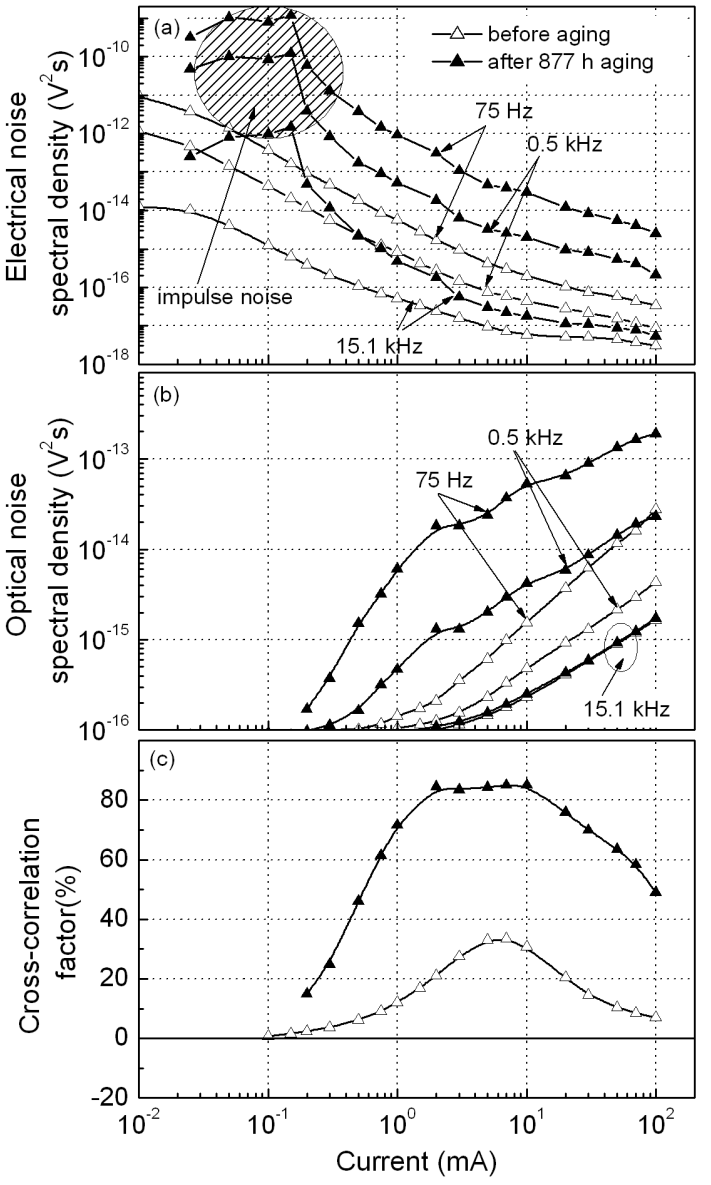

Fig. 5. Blue LED electrical (a) and optical (b) noise spectral densities at three frequencies $(75 \mathrm{~Hz}, 0.5 \mathrm{kHz}$, and $15.1 \mathrm{kHz}$ ) and simultaneous cross-correlation factor (c) (frequency range from $10 \mathrm{~Hz}$ to $20 \mathrm{kHz}$ ) dependences on current before (open symbols) and after $877 \mathrm{~h}$ (solid symbols) aging.

is mainly caused by the generation-recombination processes and at low current region (below $0.1 \mathrm{~mA}$ ) its flow is not even through the whole device cross-section - influence of defects formed current flow channels shows up. Current deviation from exponential law at currents above $10 \mathrm{~mA}$ is due to the series resistance of LED.

During aging $I-V$ characteristics of investigated LEDs strongly change (Fig. 3): at small current region (below $0.1 \mathrm{~mA}$ ) the influence of leakage current due to additional defects produced during aging strongly increases. The existence of such defects not only increases the leakage current, but also leads to the increase of nonradiative recombination: at small currents $(0.1-1 \mathrm{~mA})$ the light output power decreases about 50\% (Fig. 4). These leakage currents have almost no influence to the LED optical power characteristics at larger currents: in the current range from $1 \mathrm{~mA}$ to $10 \mathrm{~mA}$ the light output power after short time aging increases about $24 \%$ and stays stable during further aging; at currents above $10 \mathrm{~mA}$ there is no remarkable change of output power observed. 

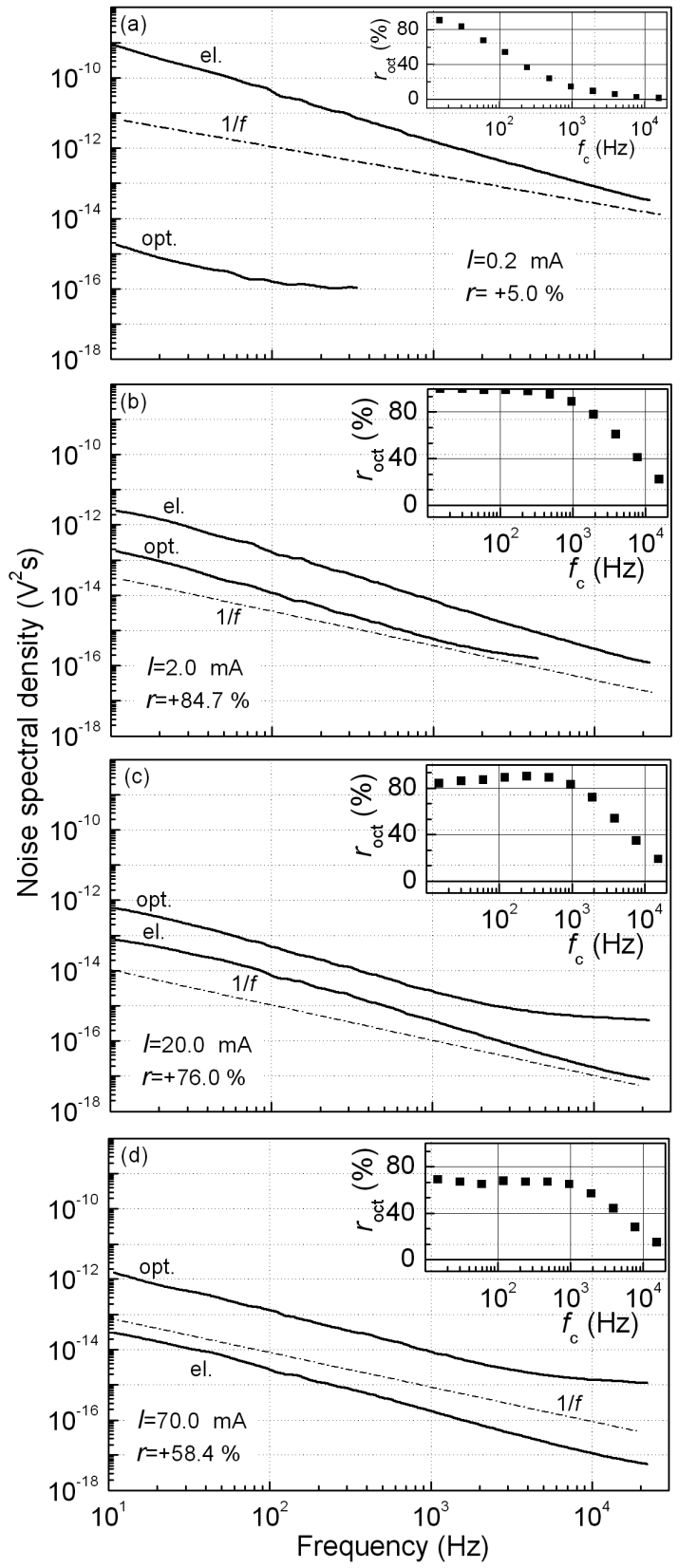

Fig. 6. Optical (opt.) and electrical (el.) noise spectra at different currents $(I)$ after $877 \mathrm{~h}$ aging $(r$ is averaged cross-correlation factor in frequency range from $10 \mathrm{~Hz}$ to $20 \mathrm{kHz}$ ), and cross-correlation factor between optical and electrical fluctuations in one-octave frequency band $r_{\text {oct }}$ dependence on central frequency $f_{\mathrm{c}}$ of octave filter is presented in insets.

$1 / f^{\alpha}$-type electrical and optical noise (that dominates at frequencies lower than $1 \mathrm{kHz}$ ) intensity of the investigated blue LEDs increases gradually with aging time increasing in the all investigated current region (Figs. 5 and 6): electrical noise spectral density after $877 \mathrm{~h}$ aging is $2-3$ orders of magnitude larger comparing to the results before aging, optical noise spectral density increases about $1-2$ orders of magnitude during the same aging. As stated above, $1 / f^{\alpha}$-type noise is due to charge carrier generation-recombination in defect centers. Therefore, $1 / f^{\alpha}$-type noise intensity increase indicates that number of defects during aging increases in the device. The shot noise is caused by random photons emission and its level depends only on the emitted light power. The observed simultaneous cross-correlation factor between optical and electrical fluctuations is positive, its value at currents smaller than $10 \mathrm{~mA}$ increases with forward current increase (graph (c) in Fig. 5). At small currents the correlation factor decreases due to larger contribution to total optical noise of thermal noise of photodetector load resistance, and at currents larger than $10 \mathrm{~mA}$ correlation coefficient decreases due to increase of emitted light shot noise, because thermal and shot noises of optical channel are not correlated with the noises of the electrical channel. Noise characteristic changes during aging show that investigated blue LED degradation is caused by defect number increase in the structure. The increase of the simultaneous cross-correlation factor between optical and electrical fluctuations during aging (graph (c) in Fig. 5)) shows that these defects are mainly created in the active region, and that GR processes in these defects modulate that part of charge carrier which recombine in the active region, and therefore, modulate emitted output light power.

Cross-correlation factor dependence on the central frequency $f_{\mathrm{c}}$ of octave filter (insets in Fig. 6) also show that strongly correlated are only the noise components at low frequencies (i.e. where $1 / f^{\alpha}$-type noise dominates (Fig. 6)). Additionally, after $877 \mathrm{~h}$ aging pulse type electrical noise appeared at low current $(0.03-0.2 \mathrm{~mA})$ region (lined area in graph (a) in Fig. 5). This noise appears as series of short pulses. Therefore electrical noise measurement results at very small currents give additional valuable information on processes in LEDs. We suppose that investigated LED degradation during accelerated aging leads to the formation defects in the active region or near its interfaces. Appearance of such defects is also a reason of lower light output power after aging in current region from $0.1 \mathrm{~mA}$ to $1 \mathrm{~mA}$ (Fig. 4) as it causes the increase of leakage current and nonradiative recombination.

Considering that shot noise and noises of measurement systems are not correlated with other noise sources, we can estimate cross-correlation factor in every one-octave frequency band, including only the low-frequency $(1 / f$, $1 / f^{\alpha}$ and Lorentzian type) noise components

$$
r_{\text {lf oct }}=\left\langle u_{\text {el oct }}(t) u_{\text {ph oct }}(t)\right\rangle /\left(\sigma_{\text {el lf oct }}^{2} \sigma_{\text {ph lf oct }}^{2}\right)^{1 / 2},
$$

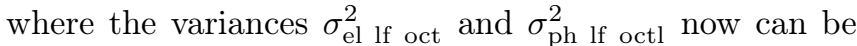
estimated from the experimental spectra of noises. The calculation results of cross-correlation factor $r_{\text {lf oct }}$ show that at currents smaller than $10 \mathrm{~mA}$ electrical and optical noises are completely correlated in all one-octave frequency bands, but at larger currents cross-correlation factor decreases with forward current increase. The lat- 
ter results show that at larger currents the electrical noise has low-frequency noise components which are due to defects in passive regions of LED structure, and these defects have very small effect to light power fluctuations.

\subsection{Aging process in AlInGaP red LEDs}

Current-voltage $(I-V)$ and light output power vs. current $(L-I)$ characteristics of AlInGaP red LEDs are presented in Figs. 7 and 8, respectively. The non-ideality factor $n$ of the $I-V$ characteristic is about 1.4, which shows on large contribution to the current of diffusion component in these samples. At currents lower than $0.3 \mu \mathrm{A} I-V$ characteristic is determined by current flow through the channels formed by defects, but not through the whole device cross-section. There are no changes of investigated LED $I-V$ characteristics during aging (Fig. 7). Also, at operation currents larger than $1 \mathrm{~mA}$ the $L-I$ characteristic has no changes during aging (Fig. 8). At smaller currents $(<0.1 \mathrm{~mA})$ the light output power of investigated LEDs increases about $40 \%$ after $2400 \mathrm{~h}$ of aging.

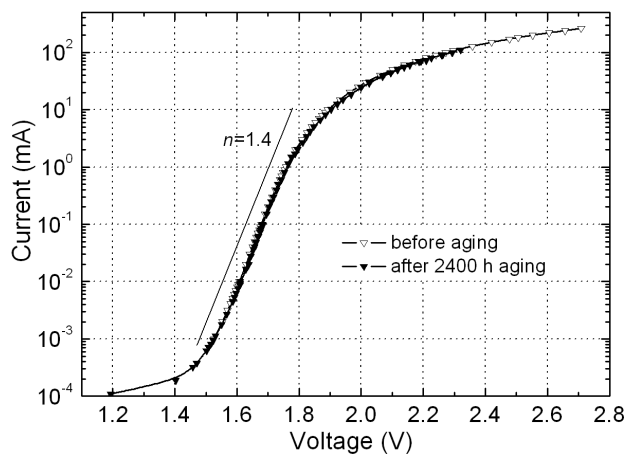

Fig. 7. Typical current-voltage characteristics of red LED before (open symbols) and after $2400 \mathrm{~h}$ (solid symbols) aging.

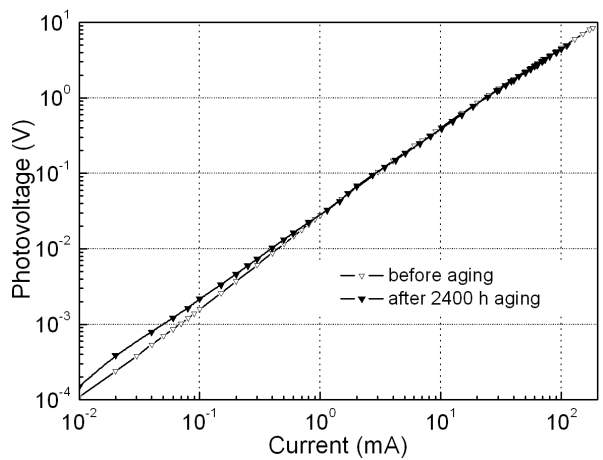

Fig. 8. Typical light output power vs. current characteristics of red LED before (open symbols) and after $2400 \mathrm{~h}$ (solid symbols) aging (light output power is proportional to the voltage of photodetector load resistance).

Investigated red LEDs before aging demonstrate quite intensive electrical noise at current region from $1 \mathrm{~mA}$ to
$100 \mathrm{~mA}$ (graph (a) in Fig. 9) that is slightly negatively (cross-correlation value is less than 10\%) correlated with optical fluctuations (graph (c) in Fig. 9). Thus, it weakly influences the light-emitting process in the device active region: major part of the injected carriers recombine nonradiatively outside the active region; there are defects in passive layers of LED structure that mainly cause the electrical noise characteristics.

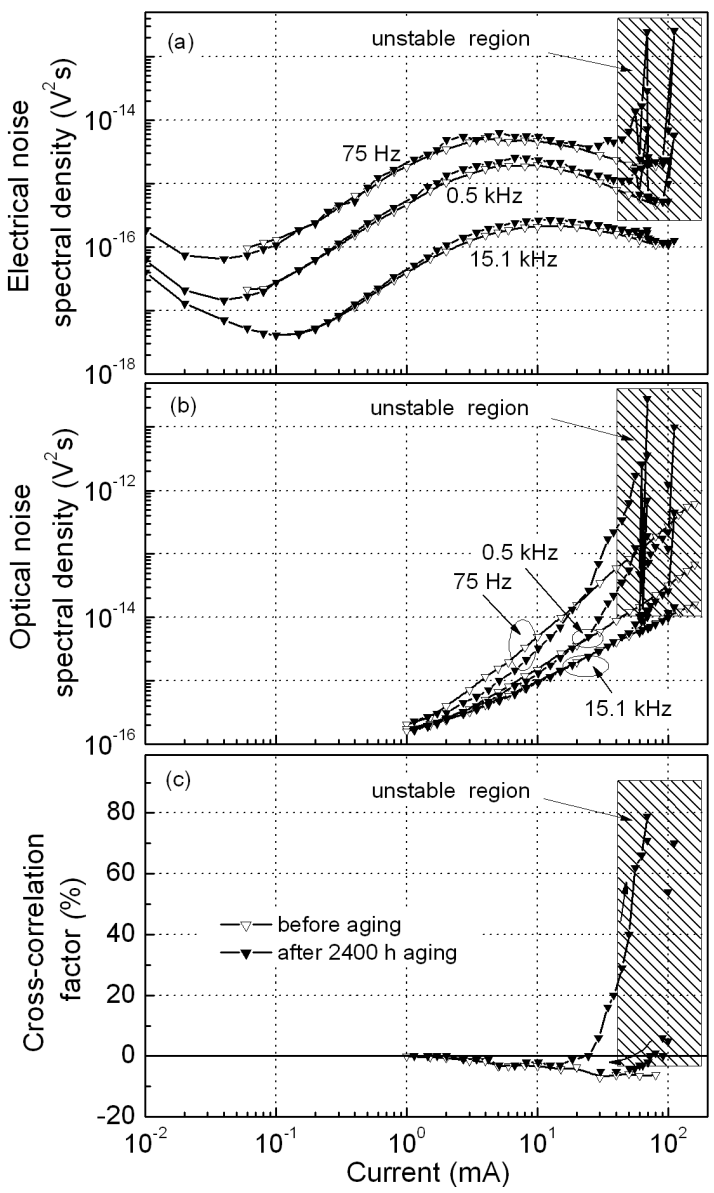

Fig. 9. Red LED electrical (a) and optical (b) noise spectral density at three different frequencies $(75 \mathrm{~Hz}$, $0.5 \mathrm{kHz}$, and $15.1 \mathrm{kHz}$ ) and cross-correlation factor (c) (frequency range from $10 \mathrm{~Hz}$ to $20 \mathrm{kHz}$ ) dependences on current before (open symbols) and after $2400 \mathrm{~h}$ (solid symbols) aging.

Noise characteristics of investigated LEDs have shown small changes during almost $2000 \mathrm{~h}$ aging: electrical noise intensity during this time of aging only at small currents $(<0.2 \mathrm{~mA})$ slightly decreased, the low-frequency (at $75 \mathrm{~Hz}$ ) optical noise level for investigated LEDs after $2400 \mathrm{~h}$ aging at medium operation currents from $3 \mathrm{~mA}$ to $20 \mathrm{~mA}$ decreases about 1.5-2 times. However after approximately $2400 \mathrm{~h}$ aging by $350 \mathrm{~mA}$ current some of investigated red LEDs began to demonstrate unstable operation. Optical and electrical noise intensity started to increase rapidly with forward current at current larger than $20 \mathrm{~mA}$ (Fig. 9). Also positive cross-correlation fac- 


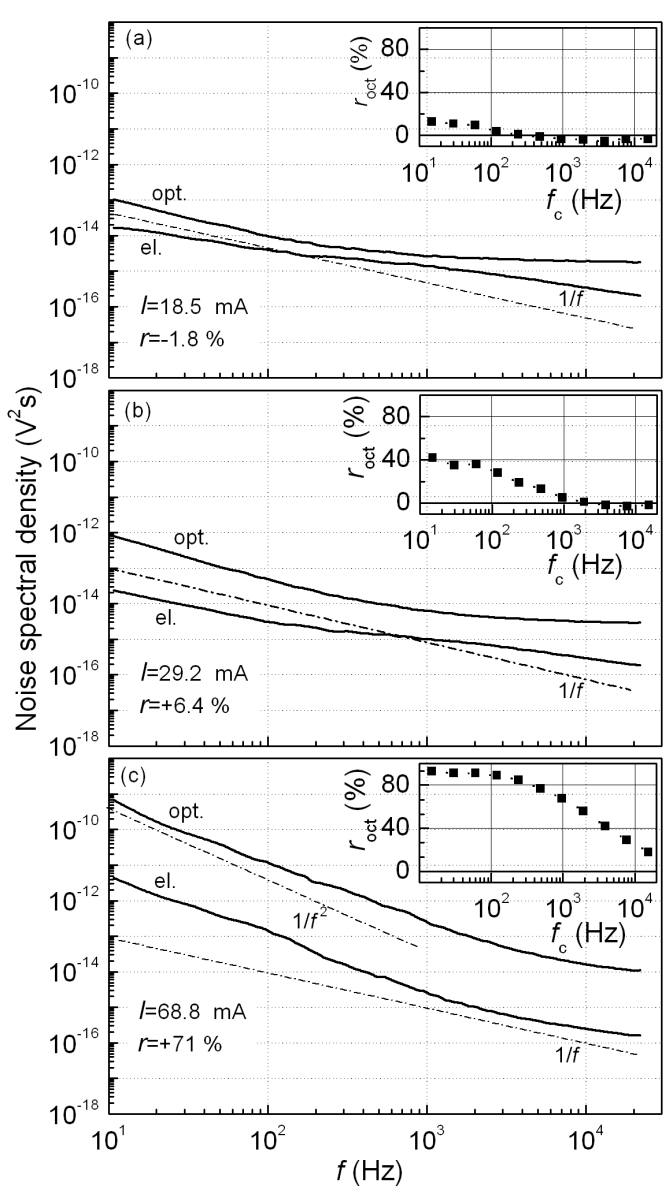

Fig. 10. Optical (opt.) and electrical (el.) noise spectra for the red LED (unstable after aging) at different currents $(I)$ after $2400 \mathrm{~h}$ aging, and cross-correlation factor between optical and electrical fluctuations in one-octave frequency band $r_{\text {oct }}$ dependence on central frequency $f_{\mathrm{c}}$ of octave filter (in insets).

tor between optical and electrical fluctuations appeared (graph (c) in Fig. 9). Large electrical and optical noise intensity increase is related only with $1 / f^{\alpha}$-type noise (Fig. 10). In current range from $25 \mathrm{~mA}$ to $100 \mathrm{~mA}$ the noise level is very unstable (Fig. 9, lined region): there were no repeatable noise measurement results by increasing and decreasing the current. Even after $2400 \mathrm{~h}$ aging the average light output power in this current range was approximately the same as before aging (Fig. 8). Optical and electrical noise spectra after $2400 \mathrm{~h}$ aging at small currents $(<20 \mathrm{~mA})$ are similar to that before aging (graph (a) in Fig. 10): electrical noise spectrum is $1 / f^{\alpha_{-}}$ -type, optical noise spectra contain $1 / f^{\alpha}$-type (where $\alpha$ is close to 1) and shot noise components, and cross-correlation factor over measured frequency band and in every one-octave frequency band (in insets) is small. At larger currents the level of electrical and optical fluctuations, and positive cross-correlation factor at low frequencies $(<1 \mathrm{kHz})$ extremely increase (graphs (b) and (c) in Fig. 10), but these intensive electrical and optical noises with $1 / f^{\alpha}$-type spectra (where $\alpha$ is close to 2 ) are unstable: they are different when current is increased or decreased. As shown in inset of Fig. 10 (graph (c)) these electrical and optical fluctuations with $1 / f^{2}$-type spectrum are strongly correlated.

Such not repeating LED operation indicates that during long-time aging in some device structure unstable defects appear. Large correlation between optical and electrical noises shows that these defects are produced in the LED active region or in its interfaces. The observed optical and electrical noise spectra type is characteristic for random telegraph processes in capture centers with large relaxation time. These defects act as nonradiative recombination centers that modulate charge carrier number in the active region and, therefore, emitted light power.

So, some of investigated red LEDs after $2400 \mathrm{~h}$ aging at maximum permissible forward current $(350 \mathrm{~mA})$ at room temperature became unstable due to unstable defects appearing in the active region or its interfaces, that act as random telegraph processes and increase nonradiative recombination.

\section{Conclusions}

A comprehensive investigation of phosphide-based red and nitride-based blue LEDs characteristic changes during aging has been carried out. The large low-frequency electrical noise level indicates that in the LED structure there are many defects and imperfections. But cross-correlation factor between optical and electrical fluctuations analysis has shown that large level of electrical fluctuations is not necessarily related with defectiveness of the LED active region, it could be related with the charge carrier recombination and capture processes in defects outside the active region, and weakly influences the emitted light. Nevertheless, these defects lead to the large leakage current out of the active region and, therefore, lower light output power.

Degradation for some of investigated LEDs is caused by the unstable defects appearance in the active region or on its interfaces. The presence of such defects reflects in the noise characteristics as additional high intensity random telegraph noise or intensive strongly correlated optical and electrical fluctuations with $1 / f^{2}$-type spectrum that is characteristic for generation-recombination processes. These defects strongly increase the leakage current, act as nonradiative recombination centers and modulate the charge carrier number capture in the active region, too, emitted photon number. This degradation mechanism is common for both phosphide-based and nitride-based investigated LEDs.

On the ground of obtained experimental results and calculation data we can state that the correlated part of electrical fluctuations for investigated LEDs is related with the defect presence in the active layer and its interfaces, while the uncorrelated part of electrical noise is related with the defects in passive regions of the LED structure. 
Investigation results have shown that noise characteristic measurement is more sensitive indicator to the LEDs degradation: noticeable changes in noise characteristics can be observed earlier and manifest stronger than they appear in static (light output power vs. current) LEDs characteristics. Thus, investigation of electrical and optical noises and their cross-correlation for LEDs reveals the physical processes that take place in device structure and material, and gives valuable information for improving device design and technological process, and allows device lifetime prediction.

\section{References}

[1] Z. Li, P.T. Lai, H.W. Choi, IEEE Photon. Technol. Lett. 21, 1429 (2009).

[2] K.-M. Chang, J.-Y. Chu, C.-C. Cheng, IEEE Photon. Technol. Lett. 16, 1807 (2004).

[3] S.J. Chang, L.W. Wu, Y.K. Su, Y.P. Hsu, W.C. Lai, J.M. Tsai, J.K. Sheu, C.T. Lee, IEEE Photon. Technol. Lett. 16, 1447 (2004).

[4] T. Mukai, IEEE J. Select. Topics Quant. Electron. 8, 264 (2002).

[5] M. Koike, N. Shibata, H. Kato, Y. Takahashi, IEEE J. Select. Topics Quant. Electron. 8, 271 (2002).
[6] J. Berntgen, T. Lieske, B. Schineller, M. Deufel, M. Heuken, H. Juergensen, K. Heime, in: Proc. 10th Int. Conf. on Indium Phosphide and Related Materials, Tsukuba 1998, p. 741.

[7] V. Palenskis, Lith. Phys. J. 30, 107 (1990).

[8] D. Ursutiu, B.K. Jones, Semicond. Sci. Technol. 11, 1133 (1996).

[9] S. Pralgauskaite, V. Palenskis, J. Matukas, Fluct. Noise Lett. 4, L365 (2004).

[10] S. Pralgauskaite, V. Palenskis, J. Matukas, J. Petrulis, G. Kurilčik, Fluct. Noise Lett. 7, L367 (2007).

[11] B. Šaulys, J. Matukas, V. Palenskis, S. Pralgauskaitè, in: Proc. Conf. on Microwave Radar and Wireless Communications (MIKON), Ed. B. Levitas, Geozondas, Vilnius 2010.

[12] G. Letal, S. Smetona, R. Mallard, J. Matukas, V. Palenskis, S. Pralgauskaite, J. Vac. Sci. Technol. A 20, 1061 (2002).

[13] V. Palenskis, J. Matukas, S. Pralgauskaitè, B. Šaulys, Fluct. Noise Lett. 9, 179 (2010). 